\section{$\underset{\substack{\text { hommes } \\ \text { \& migrations }}}{ }$}

\section{Hommes \& migrations}

Revue française de référence sur les dynamiques

migratoires

\section{$1279 \mid 2009$}

L'Afrique en mouvement

\title{
De la malle familiale au musée national
}

Le dernier voyage d'un énigmatique costume roumain

\section{Fabrice Grognet}

\section{(2) OpenEdition \\ 1 Journals}

Édition électronique

URL : http://journals.openedition.org/hommesmigrations/348

DOI : 10.4000/hommesmigrations.348

ISSN : 2262-3353

Éditeur

Musée national de l'histoire de l'immigration

\section{Édition imprimée}

Date de publication : 1 mai 2009

Pagination : 169

ISSN : 1142-852X

\section{Référence électronique}

Fabrice Grognet, « De la malle familiale au musée national », Hommes \& migrations [En ligne], 1279 | 2009, mis en ligne le 29 mai 2013, consulté le 22 septembre 2020. URL : http://

journals.openedition.org/hommesmigrations/348; DOI : https://doi.org/10.4000/hommesmigrations. 348

Ce document a été généré automatiquement le 22 septembre 2020.

Tous droits réservés 


\title{
De la malle familiale au musée national
}

Le dernier voyage d'un énigmatique costume roumain

\author{
Fabrice Grognet
}

\section{La dépositaire d'une mémoire familiale}

1 Le 25 mars dernier, Christine est venue remettre au musée de la Cité nationale de l'histoire de l'immigration un costume de femme roumaine ayant appartenu à son arrière-grand-mère arrivée à Paris en 1887 : "Je me suis rendue ici une première fois, puis j'y suis retournée à l'occasion d'une visite guidée. C'est à la suite de cette deuxième visite, en voyant les objets qui étaient exposés, que j'ai pensé effectuer un don. Je me suis alors souvenue que j'avais pas mal d'objets, parfois à la cave, et qu'ils risquaient de s'abîmer. Et puis finalement, ces objets ne servent plus à personne. L'histoire de la famille commence un peu à passer. J'ai deux fils et deux neveux. Je ne sais pas si ça les intéresse particulièrement. J'ai tout de même parlé de ma démarche à mon frère.

2 Dans la famille, ce qui est drôle, c'est que c'est moi qui suis dépositaire de notre histoire. Mon frère me dit toujours: "Mais d'où tu sais ça?" Je ne sais pas. Je crois que mes parents - et en particulier mon père - m'en ont plus parlé qu'ils ne l'ont fait avec mon frère. Ou cela ne l'intéressait pas, à l'époque. Enfin, je ne sais pas pourquoi, mais je m'aperçois que j'ai ce rôle de dépositaire dans la famille. J'ai le devoir d'informer mes fils et la génération suivante sur l'histoire de la famille. C'est comme ça. Et puis mes arrière-grands-parents n'ont eu qu'une fille, mes grands-parents n'ont eu qu'un fils. Tout cela fait aussi que rien n'a été dispersé dans la famille. Tout est chez moi."

\section{Odessa - Jassy - Paris}

Née à Odessa (actuelle Ukraine) en 1865, Dorothée Bick, se marie avec Paul Dulberger, quant à lui natif de Jassy (actuelle Roumanie), ville dans laquelle ils s'installent: “ Comment se sont-ils rencontrés? Je n'en ai aucune idée. Je ne connais pas grand-chose sur leur 
histoire. Tout ce que je sais, c'est qu'ils sont venus à Paris en 1887, quelques mois après la naissance à Jassy de Zéphirine, comme le Zéphire, ma grand-mère paternelle. C'étaient des gens qui avaient une certaine fortune. Mon arrière-grand-père était diamantaire et ils avaient un train de vie comportant un certain luxe. Je le vois à toutes les choses et photographies dont j'ai hérité. D'autre part, je le vois aussi au travers de la correspondance de ma grand-mère avec des cousines d'Odessa et de Bucarest, qui évoquent leurs toilettes et voyages à Monte-Carlo, à Paris, en Allemagne et en Suisse. Une cousine, Cécile, fait également des études de médecine à Würzburg, et ceci vers 1910. Les cartes postales que reçoit Zéphirine sont écrites en parfait français, ce qui me fait aussi penser que mes arrière-grands-parents parlaient déjà la langue avant de partir pour la France.

Je pense qu'ils ont quitté Jassy à cause des pogroms. Face à la discrimination qui touchait les Juifs, dès qu'ils l'ont pu, ils sont partis. Pour eux, Paris devait être la capitale du siècle des Lumières, des droits de l'homme. C'était là où on s'imaginait une liberté possible. Ce qui d'ailleurs était vrai, car ils ont très bien vécu à Paris. Ils se sont installés dans un appartement dans le IXe arrondissement. Ils devaient sûrement connaître des familles qui étaient parties avant eux, mais sans savoir concrètement ce qui les attendait. Mon arrière-grand-père étant également francmaçon, cela leur a peut-être procuré des relations et des facilités pour s'installer. Toujours est-il qu'ils devaient connaître des gens, car j'ai récupéré un service de soixante assiettes !"

Etabli à Paris, Paul, l'arrière-grand-père, continue son travail de diamantaire : "Paul n'était pas religieux, mais Dorothée l'était. Pour mon arrière-grand-père, la chose la plus importante était la connaissance. Il exhortait mon père - son petit-fils - à l'activité intellectuelle, à la maîtrise de la culture. C'était très important pour lui, je l'ai retrouvé dans leur correspondance où il s'exprimait avec beaucoup de tendresse et d'humour."

\section{De l'Empire russe à la vie parisienne}

5 Les années passent et Zéphirine se marie à son tour, “à un Juif bien sûr, il ne pouvait en être autrement à cette époque". Sigmund (Sigismond en français), celui qui allait devenir le grand-père de Christine, vient en 1906 de Riga (Empire russe de l'époque) : "De ce côté de la famille, ils étaient pharmaciens depuis des générations, mais j'ai entendu dire que le père de Sigmund avait quant à lui des meuneries à Riga. J'ai retrouvé des traces de pharmacies à Mitau, en Lettonie et à Hungerburg, à côté de Narva, à l'est de Tallin, qui s'appelait à l'époque Reval, en Estonie. Hungerburg était alors le port de Narva et une station thermale. C'était là que le frère aîné, Sascha, avait ouvert une pharmacie. Ce qui est extraordinaire, c'est que j'ai encore un mortier de pharmacien à la maison. C'est la seule chose qui me reste de ce côté de la famille. Ça m'amuse toujours de voir que cela a franchi les générations. Mon père disait que sa famille venait du duché de Courlande. On ne parlait ni de Lettonie, ni d'Estonie, à l'époque.

6 Il disait aussi que la famille de Sigmund appartenait à une 'guilde'. La guilde était, en Russie tsariste, une corporation de riches marchands ayant leurs propres droits. Les guildes étaient depuis Pierre le Grand transmissibles héréditairement. Cela permettait à la famille de vivre comme des citoyens du tsar non-juifs. Il n'étaient pas obligés de résider dans un ghetto et $n$ 'étaient pas soumis à des restrictions. En particulier, ils pouvaient se déplacer comme ils le désiraient. Mais en cette fin de siècle, le tsar avait aboli quelque-uns de ces privilèges. Cela touchait en particulier le service militaire qui avait été allongé à six ans pour les Juifs. Les deux derniers fils de la famille ont donc été en quelque sorte obligés d'émigrer.

7 Mon grand-père est arrivé à Paris, en passant par Anvers avec son frère, Nicolas. Ce dernier avait inventé un système de conservation du blé pour l'exportation - c'est un très vieux cousin 
de mon père, un des fils de Nicolas, auquel j'ai rendu visite récemment qui m'a raconté ça - et ils sont arrivés en vivant sur un grand pied à Paris, grâce à ce brevet. Ensuite, les deux frères ont créé une industrie de parapluies et de cannes, à Oyonnax.

Ils avaient aussi des magasins de vente à Paris, boulevard Saint-Michel et rue de Rivoli. Si ma grand-mère était déjà une 'Parisienne', puisqu'elle y est arrivée âgée de quelques mois, mon grand-père restait Russe, aussi bien dans ses habitudes que dans ses méthodes d'éducation autoritaires. Mon père, quant à lui, né à Paris, était tout à fait Parisien, et il en a conservé toute sa vie l'allure et l'esprit. Quand mon grand-père est tombé malade, il a cédé ses parts de l'usine de parapluies à son frère. Mon père travaillait chez un fabricant de montres suisses. La famille a commencé à restreindre son train de vie. Et puis il y a eu la guerre..."

\section{L'énigmatique "témoin" de la venue d'une famille}

8 C'est donc au regard des différents documents, lettres et objets, dont elle a hérité et qui constituent finalement la généalogie matérielle de la famille, que Christine a choisi, pour évoquer la venue en France des membres de sa famille, de donner le costume que son arrière-grand-mère, Dorothée, avait placé dans ses bagages : "Ce costume a toujours été chez nous, dans des malles. Mon frère n'en voulait pas au moment où on a fait le tri dans les affaires des arrière-grands-parents et grands-parents et j'en ai finalement hérité."

9 La transmission tacite des objets et documents de famille n'implique pas nécessairement en effet que l'ensemble des choses héritées soit conservé. Entre les objets que l'on garde, ceux que l'on transmet, ceux dont on se défait ou que l'on jette, et encore ceux que l'on perd et que l'on regrette, on s'aperçoit que préserver les objets légués par les ascendants ne va pas forcément de soi. Et à plus forte raison les faire parler par le jeu des souvenirs et de l'oubli à l'œuvre dans la mémoire familiale.

Toujours est-il qu'après la visite du musée, ce costume prend une autre dimension, un nouveau sens. Il devient potentiellement le support d'une mémoire et le symbole de la venue en France de la famille :

"À la maison, on disait toujours: 'C'est le costume de roumaine de l'arrière grand-mère.' À mon avis, il était 'folklorique' par rapport à leurs manières de vivre. Ils ne s'habillaient pas comme cela, on le voit bien sur les photographies. Et je suppose que Dorothée ne l'a jamais porté une fois à Paris."

Dès lors que venait faire ce costume dans les bagages? Christine avance une hypothèse au regard de sa propre histoire : "J'ai épousé un Bavarois. Et quand j'ai été pour la première fois en Bavière, mon mari m'a offert un 'dirndl', un costume folklorique avec un petit corselet et un tablier que l'on porte encore souvent en Bavière et en Autriche. Quand on veut se faire beau, hommes et femmes portent volontiers le costume traditionnel. Je ne l'ai jamais mis, mais cela m'a fait plaisir et il est toujours dans mon armoire. Alors, je ne sais pas. Peut-être qu'en épousant un Roumain, mon arrière-grand-mère s'est vue, elle aussi, offrir ce costume de Roumaine? Alors, peut-être y avait-il des moments où mon arrière-grand-mère - jeune, vue la taille de guêpe de la jupe - portait ce costume. Enfin, c'est ce que j'imagine. En tous cas, elle l'a pris dans ses bagages. " Objet finalement mystérieux, évoquant une altérité culturelle et sociale, ce costume de paysanne roumaine devient le témoin matériel qui favorise la mise en récit du roman familial transmis au fil des générations. Sorti d'une malle dans laquelle il avait dormi durant de longues années, il est en définitive élu pour rendre hommage, dans la solennité du musée, aux arrière-grands-parents primo-arrivants en France : “Je n'ai pas pensé à d'autres musées pour ce don. C'était clair que c'était à la Cité que je voulais donner ce 
costume :

c'est l'immigration qui est importante à raconter au regard de ce costume. Le fait que ces gens soient arrivés, ici, en France, un jour. Qu'ils se soient installés et que, maintenant, cela fait cinq générations et bientôt six!"

\section{RÉSUMÉS}

“J'étais curieuse de voir la Cité nationale de l'histoire de l'immigration parce que c'est un thème qui m'intéresse à double titre. Tout d'abord vis-à-vis de ma famille, mais aussi parce que j'ai moimême émigré, si l'on peut dire, par mariage. Bien entendu, ce n'est pas la même immigration. Cependant, on ne se rend pas réellement compte de ce que c'est que d'arriver dans un pays étranger. Je suis partie en Allemagne de manière volontaire, ce n'était pas quelque chose de subi. Cela dit, en Allemagne, rien n'était évident. Je ne parlais pas la langue, je perdais mes références, mes critères de jugement, mes certitudes et mon humour. Et puis, vu mes origines, je ne pouvais écarter l'histoire du Troisième Reich. Mais je ne suis pas venue vous raconter mon histoire mais celle de la famille." Christine H., arrière-petite-fille de Dorothée et Paul Dulberger.

\section{AUTEUR}

\section{FABRICE GROGNET}

Ethnologue, chargé de mission au musée national de l'Histoire et des Cultures de l'immigration, $\mathrm{CNHI}$ 